\title{
Unraveling the effect of genomic structural changes in the rhesus macaque - implications for the adaptive role of inversions
}

\author{
Anna Ullastres ${ }^{1,2}$, Marta Farré ${ }^{3,4}$, Laia Capilla ${ }^{1}$ and Aurora Ruiz-Herrera ${ }^{1,3^{*}}$
}

\begin{abstract}
Background: By reshuffling genomes, structural genomic reorganizations provide genetic variation on which natural selection can work. Understanding the mechanisms underlying this process has been a long-standing question in evolutionary biology. In this context, our purpose in this study is to characterize the genomic regions involved in structural rearrangements between human and macaque genomes and determine their influence on meiotic recombination as a way to explore the adaptive role of genome shuffling in mammalian evolution.

Results: We first constructed a highly refined map of the structural rearrangements and evolutionary breakpoint regions in the human and rhesus macaque genomes based on orthologous genes and whole-genome sequence alignments. Using two different algorithms, we refined the genomic position of known rearrangements previously reported by cytogenetic approaches and described new putative micro-rearrangements (inversions and indels) in both genomes. A detailed analysis of the rhesus macaque genome showed that evolutionary breakpoints are in gene-rich regions, being enriched in GO terms related to immune system. We also identified defense-response genes within a chromosome inversion fixed in the macaque lineage, underlying the relevance of structural genomic changes in evolutionary and/or adaptation processes. Moreover, by combining in silico and experimental approaches, we studied the recombination pattern of specific chromosomes that have suffered rearrangements between human and macaque lineages.
\end{abstract}

Conclusions: Our data suggest that adaptive alleles - in this case, genes involved in the immune response - might have been favored by genome rearrangements in the macaque lineage.

Keywords: Genome shuffling, Inversions, Macaque, Recombination, Adaptation, Meiosis, Tandem repeats, Evolutionary breakpoints

\section{Background}

Large-scale genomic changes, such as inversions, translocations, fusions and fissions, contribute to the reshuffling of the genomic architecture of organisms, providing new sources of variation on which natural selection can work. In recent years, there has been an increasing number of studies focusing on the role of chromosomal reorganizations in adaptation and speciation processes [1-4], and more specifically on the influence of genome shuffling in

\footnotetext{
*Correspondence: aurora.ruizherrera@uab.cat

Institut de Biotecnologia i Biomedicina (IBB), Universitat Autònoma de Barcelona, Campus UAB, 08193, Cerdanyola del Vallès, Barcelona, Spain ${ }^{3}$ Departament de Biologia Cel.lular, Fisiologia i Immunologia, Universitat Autònoma de Barcelona, Campus UAB, 08193, Cerdanyola del Vallès, Barcelona, Spain

Full list of author information is available at the end of the article
}

recombination ([5] and references therein). In this framework, the "suppressed recombination" model has provided compelling evidence and a theoretical framework to explain how chromosome rearrangements are involved in speciation [6,7]. Under this model, reorganizations such as inversions would have a minimal influence on fitness when present in the heterokaryotype, but rather would suppress recombination between genomic regions involved in reorganization, leading to the reduction of gene flow between diverging populations. In this context, chromosomal rearrangements would act as genetic barriers, interfering in the fixation of favorable alleles and allowing for the accumulation of genetic incompatibilities [8]. As a way to test this hypothesis, subsequent studies have analyzed sequence divergence (patterns of nucleotide differentiation) between species as an indirect estimation 
of recombination $[9,10]$. High rates of sequence divergence detected in genes located at, or near, chromosomal rearrangements have often been interpreted as indirect evidence of chromosomal speciation through suppressed recombination [9-16]. However, few empirical data have focused on the relationship between evolutionary breakpoint regions (EBRs) and recombination rates. Initial studies in Drosophila described a strong reduction of recombination around inversion breakpoints and within the reorganization itself [17]. Analogous studies in mammals are scarce, and the role of evolutionary regions in recombination has just started to be elucidated $[5,14]$.

Whole-genome comparisons of distantly related mammalian species have provided the basis for establishing models that can explain genome dynamics [18-22]. In this sense, different approaches have been developed in recent years to define homologous synteny blocks (HSBs; i.e., regions where gene order has been conserved among species) and EBRs (regions where the synteny has been disrupted by chromosomal reorganizations) among mammalian genomes [19,23-26]. Such reconstructions have revealed that genomic regions implicated in structural changes which occurred during the evolution of species are not distributed randomly through the genome, but instead they are clustered in regions that are more prone to break and reorganize [19,23-26]. The fact that some chromosomal regions have been reused during mammalian chromosomal evolution questions (i) whether these regions are physically labile due to their DNA sequence and/or structural chromatin conformation, and (ii) whether they represent regions where selection against breakpoints is minimal [26]. Regarding the first assumption, previous studies on mammalian genomes have provided compelling evidence that EBRs can be linked to the presence of repetitive elements, such as transposable elements, segmental duplications and/or tandem repeats [19,25-31]. However, given the diversity of repetitive elements in EBRs, it is likely that sequence composition is not alone influencing genome instability, clamoring for the involvement of additional factors such as the state of the chromatin (i.e., open chromatin may drive chromosomal reorganizations [32]) or selective constraints. In this latter case, comparative genomic studies have shown that mammalian EBRs tend to localize in gene-dense regions [22,28,32]. But there is a long-standing debate on the mechanisms behind this wellknown phenomenon. Several lines of evidences indicate that EBRs are precisely located between genes (i.e., intergenic regions, see [32]) not necessarily affecting gene structure/function, while others have reported possible gene expression changes due to genome reshuffling (see [33]).

Given this context, the general picture of the genomic features and DNA organization of genomic regions affected by structural reorganizations is still incomplete, as is that of how genomic changes are transmitted to the offspring during the formation of germ cells and contribute to speciation. If genomic shuffling does affect evolutionary processes through the mechanical shearing at evolutionary breakpoints, how does it impact on meiotic recombination? In this sense, the analysis of the most recent human and chimpanzee recombination maps has revealed that rearranged chromosomes presented lower recombination rates than chromosomes that did not suffer any reorganization since the human-chimpanzee common ancestor [5]. Elucidating upon whether this pattern also holds for other mammalian species would have a relevant impact on our understanding of the role of genome shuffling in speciation. Here, we have analyzed the effect of genomic structural changes on genetic recombination in the rhesus macaque to understand the mechanisms underlying chromosomal evolution in mammals and determine, in the long-term, the influence of chromosomal reorganizations on meiotic recombination. To this end, we have firstly characterized the genomic regions involved in chromosomal rearrangements between human and macaque genomes. The rhesus monkey (Macaca mulatta, Tribe Papionini, Catarrhini) is a primate species widely used in both biomedicine and evolutionary studies [34-37]. All members of the Tribe Papionini (Macaca, Papio, Mandrillus and Cercocebus) are characterized by highly stable karyotypes that have been regarded to retain the ancestral Catarrhini karyotype [34,35]. Due to such characteristics, the macaque has often been used as a reliable primate out-group candidate for evolutionary studies when studying great apes. But despite its importance, little effort has been made in characterizing the genomic landscape of HSBs and EBRs in this species since the initial release of the rhesus macaque genome [32,36,37]. Here we provide a detailed genomic map of the structural rearrangements between human and macaque. We have refined the genomic position of known rearrangements previously reported by cytogenetic approaches and described new putative micro-rearrangements (inversions and indels) between human and macaque genomes. Moreover, we have analyzed the repetitive DNA content and gene density in relation to chromosomal reorganizations, as well as the effect of inversions in meiotic recombination, detecting immune-related genes in evolutionary breakpoint regions in the macaque genome.

\section{Results and discussion}

Homologous synteny blocks (HSBs) and evolutionary breakpoint regions (EBRs) in human and rhesus macaque genomes

In order to analyze the chromosomal reorganizations (fusions/fissions, translocations and inversions) between human and rhesus macaque, two different algorithms 
were applied: SyntenyTracker and Cassis (See Material and Methods for further information). Both approaches can detect HSBs and EBRs in both species based on the positions of orthologous genes. By analyzing a total of 16,133 orthologous genes between Homo sapiens (HSA) and Macaca mulatta (MMU), SyntenyTracker detected 59 EBRs in both genomes, with a median length of $259 \mathrm{Kbp}$ in the human genome (ranging from $11.6 \mathrm{Kbp}$ to 4.6 $\mathrm{Mbp}$ ) and $163.6 \mathrm{kbp}$ in the macaque (ranging from 16.9 Kbp to $6.1 \mathrm{Mbp}$ ) (Table 1 and Figure 1). Cassis, on the other hand, detected 109 EBRs in the human genome, and 111 EBRs in the macaque (Table 1), with a median length of $51.3 \mathrm{Kbp}$ for human EBRs (ranging from $3 \mathrm{bp}$ to 4.5 $\mathrm{Mbp}$ ), and $26 \mathrm{Kbp}$ for the rhesus macaque (ranging from $7 \mathrm{bp}$ to $512.5 \mathrm{Kbp}$ ) (Table 1). Merging the results obtained with both approaches resulted in 121 EBRs in the human genome and 125 EBRs in the macaque. After applying a conservative filtering process (See material and methods for further information), we obtained a total of 74 EBRs and 101 HSBs in the human genome with a median EBR length of $51.6 \mathrm{Kbp}$ (ranging from $3 \mathrm{bp}$ to $508.7 \mathrm{Kbp}$ ). Likewise, we obtained 77 EBRs and 94 HSBs in the macaque genome (Table 1 and Additional file 1: Table S1, Additional file 2: Table S2 and Additional file 3: Figure $\mathrm{S} 1$ ), with a median EBR length of $26 \mathrm{Kbp}$ (ranging from 7 bp to $420.3 \mathrm{Kbp})$.

Our own study represents a departure from those conducted previously $[27,28,30]$ in that it relies on a detailed comparison between human and rhesus macaque genomes based on orthologous genes and whole-genome sequence alignments. Previous cytogenetic studies delineated the primate ancestral karyotype, defining conserved syntenies among species and the direction of chromosomal rearrangements in a phylogenetic context [24,34,38,39]. Species from the Tribe Papionini - including Macaca, Papio, Mandrillus and Cercocebus - are characterized by sharing the same karyotype and large-scale chromosomal reorganizations since their divergence from a common primate ancestor $[34,35]$. When comparing these species with the human karyotype, previous cytogenetic studies described the presence of 20 intra- and inter-chromosomal reorganizations [34,35,37,40]. These rearrangements include 12 pericentric inversions affecting eleven chromosomes, four paracentric inversions involving four chromosomes and four fusions/fissions $[34,35,37,40]$. Overall, our in silico approach confirmed the presence of the above-mentioned macro-reorganizations and, thus, refined the breakpoints involved in both genomes (Additional file 1: Table S1). Moreover, we identified 39 and 41 previously undetected EBRs in the human and rhesus genome, respectively, affecting 13 different chromosomes (Additional file 1: Table S1). This resulted in 21 previously undetected reorganizations in the human genome and 23 in the macaque. Although previous cytogenetic studies have reported that six chromosomes (MMU6, MMU8, MMU11, MMU17 and MMU19) have been maintained collinear between both species, our results suggest that only chromosomes MMU6, MMU12 and MMU17 have maintained a complete conserved synteny. Six new indels (insertions or deletions) were also identified, ranging from $3.3 \mathrm{Kbp}$ to $2,784.8 \mathrm{Kbp}$ in five different chromosomes (Additional file 1: Table S1).

Tandem repeats do not accumulate in EBRs in the macaque genome; instead, they are correlated with the evolutionary history of chromosomes

Previous comparative genomic studies have revealed that mammalian breakpoint regions are especially rich in repetitive elements, such as segmental duplications [41-43], repetitive sequences [25,42], transposable elements and long regulatory regions $[26,29,44-46]$. We tested whether this pattern applies to the rhesus macaque, a species that has retained the ancestral Catarrhini karyotype [34,35]. To this end, the genome distribution of tandem repeats (TR) in this species was analyzed. A total of 701,128 loci, representing $60.9 \mathrm{Mbp}$ of the whole genome was detected. In order to study the genome-wide distribution of TR, the number of base pairs involved in TR by screening nonoverlapping windows of $100 \mathrm{Kbp}$ along the genome was analyzed. When considering the number of base-pairs (per $100 \mathrm{Kbp}$ ) of each window covered by TR, we observed a significant increase of TR in telomeric and centromeric regions when compared to HSBs and EBRs (Kruskal-Wallis test, p-value $<0.0001$ ), mirroring previous observations obtained in the human and great apes $[25,26]$. Subsequently, we tested whether there was a correspondence between TR and EBRs in all macaque chromosomes. Our analysis indicated that EBRs are not significantly enriched by tandem repeats when compared to HSBs (Mann-Whitney U test, p-value > 0.05).

Table 1 Summary of the evolutionary breakpoint regions (EBRs) detected in the human (HSA) and macaque (MMU) genomes

\begin{tabular}{|c|c|c|c|c|c|c|}
\hline & \multicolumn{2}{|c|}{ SyntenyTracker } & \multicolumn{2}{|c|}{ Cassis } & \multicolumn{2}{|c|}{ Merged } \\
\hline & HSA & MMU & HSA & MMU & HSA & MMU \\
\hline Number of EBRs & 59 & 59 & 109 & 111 & 74 & 77 \\
\hline Minimum length (Kbp) & 11.6 & 16.9 & 0.003 & 0.007 & 0.003 & 0.007 \\
\hline Maximum length (Kbp) & $4,614.2$ & $6,114.9$ & $4,505.9$ & 512.5 & 508.7 & 420.3 \\
\hline Median length (Kbp) & 259.3 & 163.6 & 51.3 & 26 & 51.6 & 26 \\
\hline
\end{tabular}




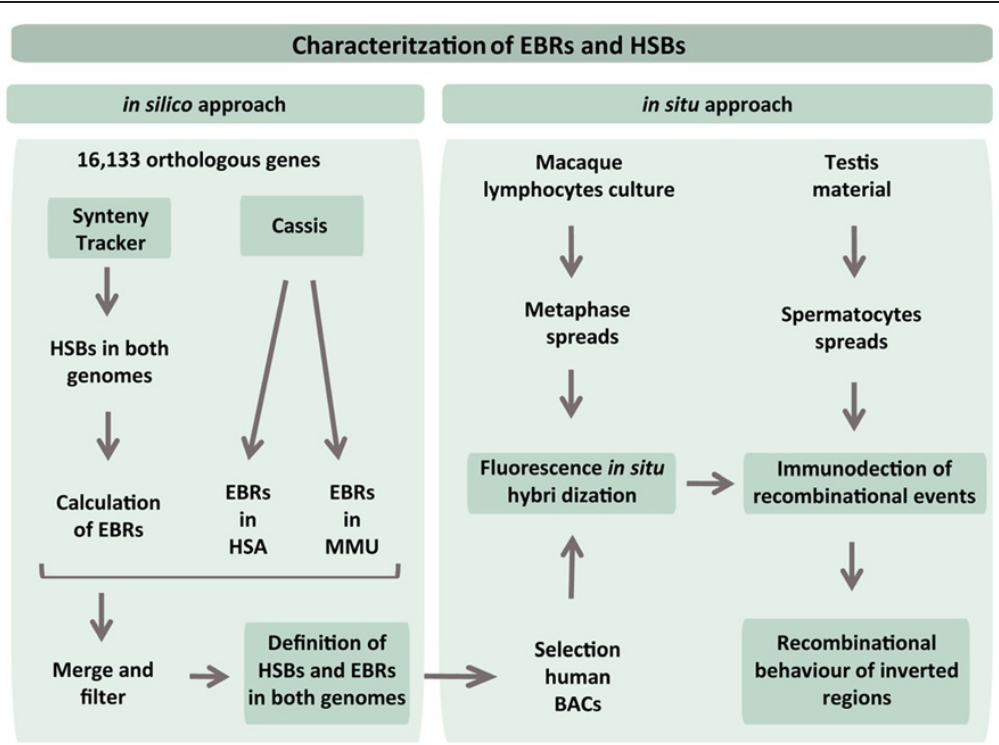

Figure 1 Experimental design of the study.

Recent studies in great apes have found differences in the genome-wide distribution of TR among species, suggesting that they might be correlated with the evolutionary history of each primate chromosome [26]. More specifically, qualitative comparisons of TR distribution in great apes indicated that the TR landscape might have been conserved in collinear chromosomes, but altered in those reorganized chromosomes [26]. Under this assumption, genomic regions that have suffered more rearrangements during their evolution are expected to concentrate more repetitive sequences than are conserved regions. In fact, our dataset supports this assumption since rearranged chromosomes in the macaque lineage (MMU3 and MMU5) have more TRs than do those that maintained the ancestral form (MMU2, MMU7, MMU10, MMU12, MMU13 and MMU18) (Mann-Whitney U test, $\mathrm{p}$-value $<0.0001)$. Despite the limitations of the current rhesus macaque draft genome assembly and annotation $[47,48]$, this view is consistent with the lack of differences found in TR density between EBRs and HSBs in the macaque, which has maintained an ancestral karyotype within Catarrhini and, consequently, can be considered to have retained a more conserved chromosome complement (i.e., low degree of genome reshuffling) than have those of great apes $[25,26]$.

\section{Defense-responsive genes are over-represented in EBRs}

Once the evolutionary genomic landscape of the macaque was established, the genome-wide distribution of genes was further examined, paying special attention to gene ontology. A total of 28,595 genes was included in the analysis: 21,023 protein-coding genes; 5,913 non-coding RNA genes and 1,659 pseudogenes. We scrutinized each macaque chromosome's complete sequence using nonoverlapping windows of $100 \mathrm{Kbp}$ in order to analyze the distribution of genes genome-wide. The mean distribution is 0.98 genes per $100 \mathrm{Kbp}$ (including protein-coding genes, non-coding RNA genes and pseudogenes) in the whole macaque genome. When analyzing the distribution of protein-coding genes in more detail, a higher gene density in EBRs was detected (1.48 genes/100 Kbp), when compared with HSBs (0.73 genes/100 Kbp) (Kruskal-Wallis test, $\mathrm{p}$-value $<0.0001)$. Therefore, our results indicate the presence of EBRs in gene-rich regions, in line with previous observations in mammals using multi-species comparative maps [22,32]. In trying to understand the reasons behind this pattern, initial studies reported the intergenic location of mammalian EBRs [22], while recent studies have paid special attention to the adaptive role of EBRs [33]. This has been the case of the pig, for example, where EBRs have been found to be especially rich in taste perception networks [49], suggesting that genome reshuffling significantly contributed to adaptation and the development of lineage-specific traits. Moreover, it has also been suggested that inversions can suppress recombination within the affected zones [5-8]. Consequently, rearranged sequences could accumulate alleles, which might be adaptive for the population, and this could generate reproductive isolation leading, eventually, to speciation.

With this in mind, the function of the genes located at or near EBRs was analyzed in the macaque genome through the analysis of GO terms enrichment. We focused our study on the genes located in the positions of the EBRs detected in silico, considering flanking regions of $\pm 200 \mathrm{Kbp}$ in size in order to cover genes that overlapped EBR starts and/or ends. We applied the Functional Annotation 
Clustering Tool from DAVID database [50] using a background list containing 19,794 protein-coding genes. This was created by filtering the total protein-coding genes list available in the database, discarding 2,111 genes located in the centromeres and telomeres. Using this approach we found that macaque EBRs are enriched in genes related to the immune system. In fact, we detected a single functional module significantly associated to EBRs (Enrichment Score $=2.81$, Table 2 and Additional file 4: Table S3). This functional module included 17 genes implicated in the immune response (including several GO terms, such as chemokine, defensin precursors and Toll-like receptor signalling pathway, among others) located in seven different macaque EBRs (Table 2, Additional file 4: Table S3). Interestingly, five of these genes are beta-defensins, and are clustered in MMU10 EBR involved in the pericentric inversion that occurred after macaque and human divergence (Table 2). Beta-defensins are antimicrobial peptides involved in the resistance to microbial colonization of the epithelial surface [51]. This cluster, whose expression is restricted to the male reproductive tract, is the result of a series of duplication events subsequently shaped by the action of positive selection [52,53]. In fact, previous studies have shown a marked differential expression of DEFB118 and DEFB122 (two of the genes detected in MMU10, Table 2) in human and macaque reproductive tissues [50]. Since chromosomal rearrangements are sources of genome variation, it might be possible that they could have influenced the structure of regulatory regions in those genes near EBRs. Moreover, and in line with these observations, we also identified an alfa-defensin cluster in the vicinities of the EBR detected in MMU8 (Table 2). This gene cluster has been previously described as an example of rapid evolution in primates [54]. Developing protection to new microbial infections is among one of the great challenges that species confront during the adaptation to new ecological niches. Thus, the generation of new defensin variants through different mechanisms, such as sequence modifications when a chromosomal reorganization occurs, could lead to adaptation to new environments. In fact, defensin clusters have also been described as being located in EBRs in Cetartiodactyla [55], suggesting the importance of genome reshuffling as an important source of new gene variants.

Moreover, we also investigated genes located within the inverted regions that are specific for the macaque linage, as they could have played an adaptive role in this species. Statistically significant enrichment was detected (Enrichment Score $\geq 1.5$ ) for defense-response genes within the paracentric inversion affecting MMU5, a chromosomal reorganization that has been fixed in the lineage leading to macaque (Table 3 and Additional file 5: Table S4). MMU5 inversion spans approximately $33 \mathrm{Mbp}$ across the centromere, involving one breakpoint located between 44.38-44.44 Mbp and the other one located between 77.5-77.9 Mbp (Table 3). Specifically, we identified two different statistically significant functional modules consisting of 13 and 6 genes, respectively - mostly coding

Table 2 Genes located in macaque EBRs

\begin{tabular}{|c|c|c|c|}
\hline Gene & Distance from EBR edges (Kbp) & EBR position (chr: start-end) & Function \\
\hline GNRHR2 & 147.5 & MMU1: $126,278,886-126,466,180^{*}$ & Gonadotropin receptor \\
\hline ADIPOQ & 156.7 & MMU2: 179,026,672-179,049,651* & Adiponectin precursor \\
\hline F7D492 & 133.6 & MMU8: 8,165,750-8,338,584 & Defensin precursors \\
\hline DEFA4 & 164.6 & & \\
\hline LOC574310 & 172.8 & & \\
\hline MSMB & 31.1 & MMU9: 46,613,935-46,645,068 & Beta-microsemino protein \\
\hline DEFB123 & 189.6 & MMU10: 33,312,300-33,313,023* & Beta-defensins \\
\hline DEFB118 & 122.5 & & \\
\hline DEFB119 & 113.7 & & \\
\hline DEFB121 & 152.7 & & \\
\hline DEFB122 & 171.5 & & \\
\hline CCL18 & 129.4 & MMU16: 31,482,483-31,663,199* & C-C motif chemokine precursors \\
\hline CCL23 & 193.7 & & \\
\hline CCL3 & 106.4 & & \\
\hline Q76LL8 & 182.8 & & \\
\hline CCL25 & 106.1 & MMU19: 7,76,0383-7,770,483 & $\mathrm{C}-\mathrm{C}$ motif chemokine precursor \\
\hline DC-SIGN & 53 & & Pathogen-recognition receptor \\
\hline
\end{tabular}

Protein-coding genes of the single functional annotation term (Enrichment Score $=2.81$ ) detected in the proximity of EBRs in the macaque genome. *macro-reorganizations; MMU - macaque chromosome. 
Table 3 Genes located in the MMU5 inversion

\begin{tabular}{|c|c|c|}
\hline Gene & Distance from EBR & EBR position (start-end) \\
\hline \multicolumn{3}{|c|}{ Term $1(E R=4.72)$} \\
\hline CXCL13 & $7,573.4$ & $44,386,928-44,442,153$ \\
\hline CXCL11 & $9,135.6$ & \\
\hline CXCL10 & $9,176.8$ & \\
\hline CXCL9 & $9,193.0$ & \\
\hline CXCL3 & $11,230.2$ & \\
\hline PPBP & $11,276.2$ & \\
\hline PF4 & $11,282.6$ & \\
\hline CXCL1 & 11,420 & \\
\hline PF4V1 & $11,439.3$ & \\
\hline P67813 & $11,555.7$ & \\
\hline ALB & $11,867.3$ & \\
\hline ODAM & $15,090.7$ & \\
\hline $\mathrm{KIT}$ & $2,815.7$ & $77,560,198-77,980,505$ \\
\hline \multicolumn{3}{|c|}{ Term $2(E R=3.52)$} \\
\hline SULT1E1 & $15,330.9$ & $44,386,928-44,442,153$ \\
\hline UGT2B4 & $17,318.6$ & $77,560,198-77,980,505$ \\
\hline UGT2A3 & $17,222.2$ & \\
\hline UGT2B33 & $17,078.8$ & \\
\hline UGT2B15 & $16,769.3$ & \\
\hline SRD5A3 & $3,533.6$ & \\
\hline
\end{tabular}

Protein-coding genes contained in the two GO terms detected in the macaque-specific pericentric inversion affecting chromosome MMU5. $\mathrm{ER}=$ Enrichment Score.

for chemokines and the UDP-Glucuronosyltransferases 2B gene family -out of a total of 181 protein-coding genes present within the rearranged region (Enrichment scores: 4.72 and 3.52, respectively) (Table 3 and Additional file 5: Table S4). Both functional modules detected include immune-response genes that might have been influenced by the chromosomal rearrangement in the rhesus monkey. Chemokines are known to play a role in the neuroinflammation process in response to infection, are present in the central nervous system (CNS) and are expressed in neurons and glial cells [56]. Moreover, there is evidence suggesting that they are also involved in neurodevelopment and neurophysiological signaling [56,57]. UDP-Glucuro nosyltransferases, on the other hand, are enzymes from the major pathway for the elimination of xenobiotics and endobiotics, and it has been suggested to play a role during speciation in the lineage leading to macaque [58].

Our observation of an over-representation of defenseresponsive genes in both EBRs and macaque-specific inversions might suggest an adaptive role of reorganizations in this species. Previous studies have reported that a small proportion of the mammalian genome, i.e., $4 \%$ in the case of the human genome, is under selective constraints, especially so for coding regions, introns and intergenic regions
[59]. This suggests that in certain regions the fitness cost is so pronounced (i.e., could be lethal or deleterious for the individual and the progeny) that rearrangements are not allowed (i.e., [60]). But it has also been shown that this constraint could be somewhat relaxed in the promoters of genes linked to the immune system, reproduction and perception [59], allowing for the generation of new variability to ensure adaptation to new environments. In light of our results, this might be the case for the genomic regions under study in the macaque. However, whether immune-related genes are directly involved in lineagespecific adaptation, as has been previously suggested for macaque [58], needs further validation.

\section{Genome reshuffling and its effect on chromosome- specific recombination landscapes}

Once the genomic structural changes were defined in the rhesus macaque, together with the genome distribution of coding-genes across evolutionary regions, we further experimentally analyzed the meiotic recombination landscape as a way to explore the adaptive role of chromosomal changes. Under the "suppressed recombination" model of chromosomal evolution, chromosome rearrangements would have a minimal influence on fitness, but would rather suppress recombination within the genomic regions affected, thus contributing to the accumulation of gene incompatibilities [2,5-9]. The analysis of the most recent human and chimpanzee recombination maps inferred from genome-wide single-nucleotide polymorphism (SNP) data revealed that the standardized recombination rate was significantly lower in rearranged rather than in collinear chromosomes [5]. In the case of rhesus macaque, chro mosome-specific recombination maps are available for very few chromosomes [61], and whether or not chromosomal reorganizations that have been fixed in the macaque lineage have affected the recombination landscape was addressed in our study.

Catarrhini monkeys, and more specifically the Tribe Papionini, are characterized by the presence of highly conserved karyotypes in terms of diploid number and chromosome homologies [62,63]. It has been described that species sharing the same karyotype (i.e., no major genome reshuffling) maintain the chromosomal distribution of meiotic crossover in conserved chromosomes on a broad scale (Mbp, the resolution provided by the in situ immunoflourescence detection of meiotic proteins) $[64,65]$. Therefore, we expect that the recombination pattern in Macaca and Cercocebus chromosomes (species that share the same karyotype and belong to the Tribe Papionini $[62,63])$ are conserved at the Mbp resolution, thus allowing us to extrapolate the results obtained in both species, as previously described $[64,65]$. In this context, and taking advantage of the EBRs detected in silico in the macaque genome, we subsequently studied the chromosomal distribution of 
meiotic crossover (COs - here exemplified as MLH1 foci) in macaque chromosomes affected by inversions since their Catarrihini common ancestor (MMU5). This was done by combining the immunostaining of meiotic proteins and fluorescence in situ hybridization (FISH) with specific BAC clones spanning the EBRs detected in our in silico scanning (Figure 2, Table 4 and Additional file 6: Table S5). The analysis of COs distribution according to centromere positions and EBR-specific BAC probes allowed us to experimentally determine the chromosome position of evolutionarily reorganized regions directly on pachytene chromosomes along chromosomal axes. Our efforts were concentrated on three chromosomes, which represented three different evolutionary states: (i) a chromosome with a pericentric inversion (CTO5/MMU5) specific for the Cercocebus and Macaca lineages; (ii) a paracentric inversion (CTO9/MMU9) that has been fixed in human and chimpanzee lineages, and therefore has been maintained collinear in the macaque lineage; and (iii) a collinear chromosome (CTO6/MMU6) that has been maintained collinear since the Catarrhini common ancestor. With this in mind, our main goal was two-fold: (i) reconstruct chromosome-specific recombination maps, and (ii) encompass the distribution of meiotic crossovers across the inverted regions in order to test whether chromosomal inversions have an effect in reducing recombination.

A total of 258 spermatocytes at pachynema was analyzed in order to obtain chromosome-specific recombination maps. We detected an average of $35.24( \pm 3.01)$ MLH1 foci per analyzed cell (Figure 2). Assuming that one MLH1 focus can be translated into 50 centimorgans (cM) in genetic length according to previous studies [61,64-66],
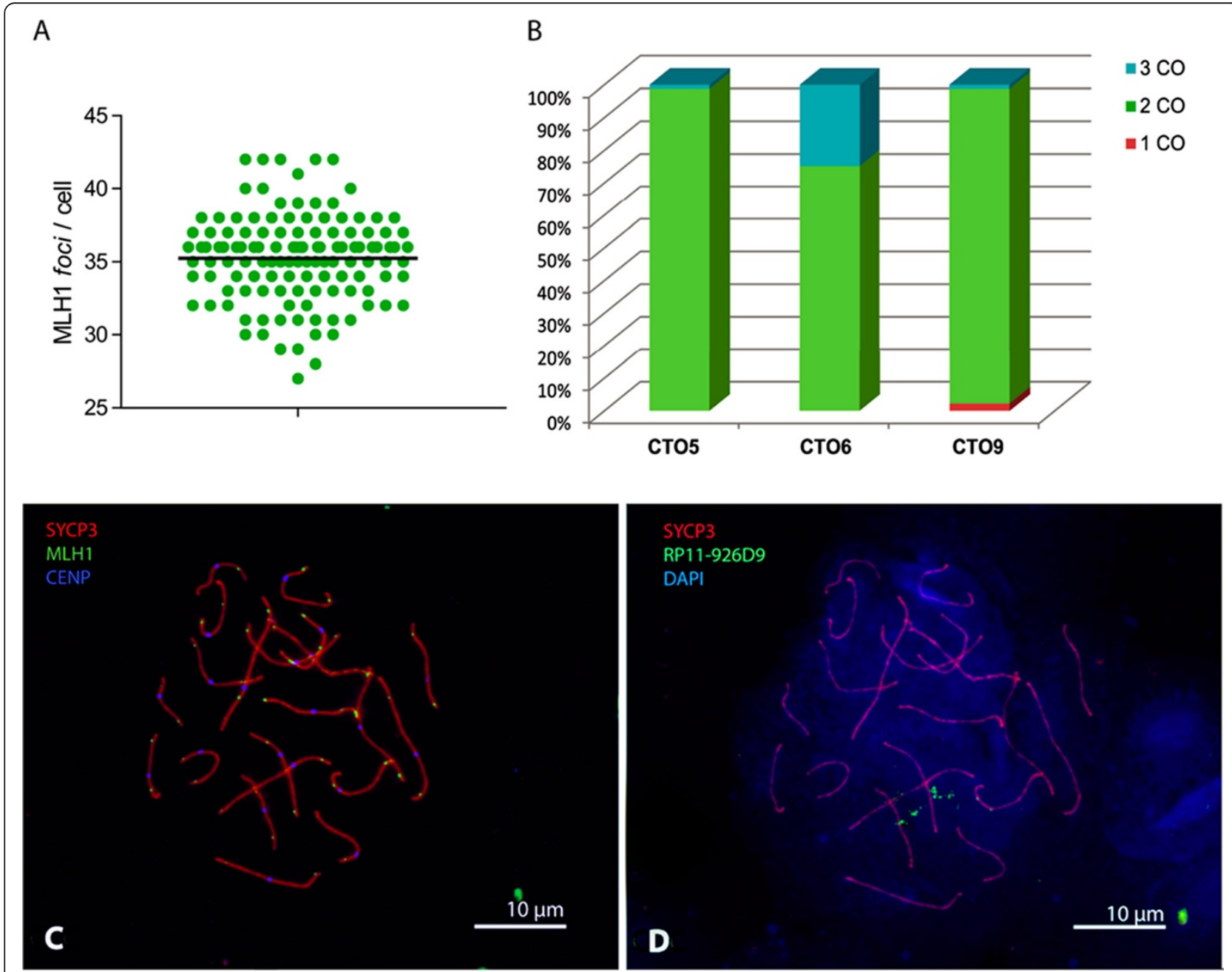

Figure 2 Recombination features in Cercocebus torquatus. (A) Number of MLH1 foci detected per cell in Cercocebus torquatus (CTO). The horizontal bar indicates the mean. (B) Comparison of the percentage of cells with a different number of MLH1 foci (Crossovers, CO) in the different chromosomes analyzed: CTO5, CTO6 and CTO9. (C-D) Sequential image of a spermatocyte at pachynema from Cercocebus torquatus depicting (C) triple immunostaining against SYCP3 (red), MLH1 (green) and centromeres (blue). (D) Representation of the same cell after applying sequential fluorescent in situ hybridization (FISH) with the BAC probe RP11-926D9, specific for CTO9. 
Table 4 Chromosome-specific recombination analysis

\begin{tabular}{|c|c|c|c|c|c|c|c|}
\hline \multirow[t]{2}{*}{ Chr } & \multirow[t]{2}{*}{$\mathrm{N}$} & \multicolumn{2}{|c|}{$\mu \mathrm{m} \mathrm{SC}$} & \multirow{2}{*}{$\begin{array}{l}\text { MLH1 } \\
\text { foci/ } \\
\text { SC }\end{array}$} & \multirow{2}{*}{$\begin{array}{c}\text { MLH1 } \\
\text { focil } \\
\mu \mathrm{m}\end{array}$} & \multicolumn{2}{|c|}{ MLH1 foci/ $\mu \mathrm{m}$} \\
\hline & & p-arm & q-arm & & & Inside inv & Outside inv \\
\hline CTO5 & 87 & 3.85 & 4.01 & 2.01 & 0.26 & 0.04 & 0.31 \\
\hline СТO6p & 85 & 2.26 & 6.12 & 2.25 & 0.27 & 0.06 & 0.30 \\
\hline CTO6q & 85 & 2.26 & 6.12 & 2.25 & 0.27 & $0.09^{\#}$ & 0.36 \\
\hline СТО9 & 86 & 3.19 & 5.56 & 1.99 & 0.23 & $0.03^{*}$ & 0.31 \\
\hline
\end{tabular}

Chr: Chromosome, CTO: Cercocebus torquatus, N: number of cells analyzed, SC: synaptonemal complex, Inv: inverted region. CTO6p: inversion simulated in the small chromosome arm. CTO6q: inversion simulated in the long chromosome arm. \# CTO6 density (expressed as MLH1 foci $/ \mu \mathrm{m}$ ) inside and outside the inversion refers to the simulated region for the comparison in each case. *indicates statistical significance ( $p$-value $<0.05$ ) when comparing reorganized region with simulated inversion in the collinear chromosome CTO6 (Mann Whitney $\mathrm{U}$ test).

these recombination events correspond to an autosomal genetic length of $1,760 \mathrm{cM}$ for $C$. torquatus, very similar to the $1,950 \mathrm{cM}$ obtained in M. mulatta [61]. Our results also indicated that all chromosome pairs present, at least, one recombination event, with an average number of 1.8 COs per homologous autosomic chromosome and 0.88 COs per autosomic arm. Moreover, a maximum of three recombination events in the large chromosomes was detected (Figure 2). In addition, for each chromosome analyzed, its total length (expressed in $\mu \mathrm{m}$ ) and the density

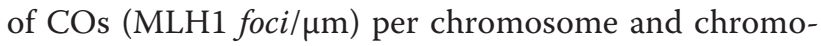
somal arm were calculated (Table 4). All three chromosomes presented equivalent $\mathrm{CO}$ densities along their synaptonemal complexes: $0.26 \mathrm{COs} / \mu \mathrm{m}$ for CTO5, 0.27 $\mathrm{COs} / \mu \mathrm{m}$ for $\mathrm{CTO} 6$ and $0.23 \mathrm{COs} / \mu \mathrm{m}$ for CTO9. In general, we detected an increase in $\mathrm{CO}$ frequency towards the chromosomal telomeric regions, whereas centromere regions showed very low $\mathrm{CO}$ frequencies (Figure 3), mirroring previous studies in mammalian species $[61,64-66]$.

Subsequently, CO density was analyzed inside and outside each inverted region using the selected BAC probes to label the location of the breakpoint in each chromosomal region affected by the inversion (Figures 2 and 3). As a general trend, we observed low $\mathrm{CO}$ densities within inverted regions $(0.04$ and $0.03 \mathrm{MLH} 1$ foci $/ \mu \mathrm{m}$ for CTO5 and CTO9, respectively), when compared to chromosomal regions outside the reorganized area $(0.31 \mathrm{COs}$ MLH1 foci per $\mu \mathrm{m}$ in both cases) in both rearranged chromosomes (Table 4). When analyzing the recombination rate within inverted regions among chromosomes, significant reduction of CO density was observed in CTO5 and CTO9 within the inverted regions, when compared with regions outside the inversion (Table 4, Mann-Whitney U test, pvalue $<0.05$ ). Such differences were not observed in the simulated inversion in CTO6, a chromosome that has been maintained collinear in the macaque lineage (Table 4).

Subsequently, we tested whether the suppression of recombination observed within reorganized areas was due to the low recombination rate characteristic of pericentromeric regions. To do so, we considered as a pericentromeric region an area extending 30\% of each chromosome arm from the centromere towards the telomeric region and compared it to the $\mathrm{CO}$ density observed in the same region in the collinear chromosome (CTO6). When all chromosomes were compared, our results showed no statistical differences among the pericentromeric regions in the small arms (CTO5p, CTO6p and CTO9p) (KruskalWallis test, $\mathrm{p}$-value $>0.05$ ); however, differences in $\mathrm{CO}$ density were significant when considering the long arms (CTO5q, CTO6q and CTO9q) (Kruskal-Wallis test, $\mathrm{p}$-value $=0.018$. Moreover, a significant reduction was observed in the $\mathrm{CO}$ density within the rearranged region in CTO9, when compared to the collinear chromosome CTO6 (Mann-Whitney U test, p-value $=0.016$ ), (Figure 3 and Table 4). These differences were not significant, however, when the inverted region of CTO5 was compared to the collinear chromosome CTO6 (Mann-Whitney U test, p-value $>0.05$ ).

What are the evolutionary implications of our observations in light of the "recombination suppression" model? Despite the fact that the "recombination suppression" was initially proposed to explain differences in recombination rates within reorganized genomics in heterokaryotypes (i.e., heterozygotes), we observed a reduction of recombination in fixed rearrangements, raising intriguing questions about the mechanisms involved. Previous studies in great apes have revealed that rearranged chromosomes presented significantly lower recombination rates than do chromosomes that have been maintained collinear since a common ancestor, and this was related to the lineage in which they become fixed [5]. Importantly, inverted regions had lower recombination rates than did collinear and non-inverted regions, independently of the effect of centromeres [5]. Although at this stage it would be premature to argue about the evolutionary forces behind this pattern, our results highlight the importance of the study of recombination framed by the evolutionary history of chromosomes and, in greater extent, genomes. Incorporating more chromosomes into the experimental study would be necessary to detect a clearer genomic effect of the inversions in the distribution of recombination patterns.

\section{Conclusions}

Genomic rearrangements might play an important role in local adaptation and species divergence by the modification of both the structure and regulation of genes located near the affected regions. Here, we provide a highly refined description of the chromosomal reorganizations and evolutionary breakpoint regions in the human and rhesus macaque genomes based on orthologous genes and genome sequence alignments. The high-resolution map of 


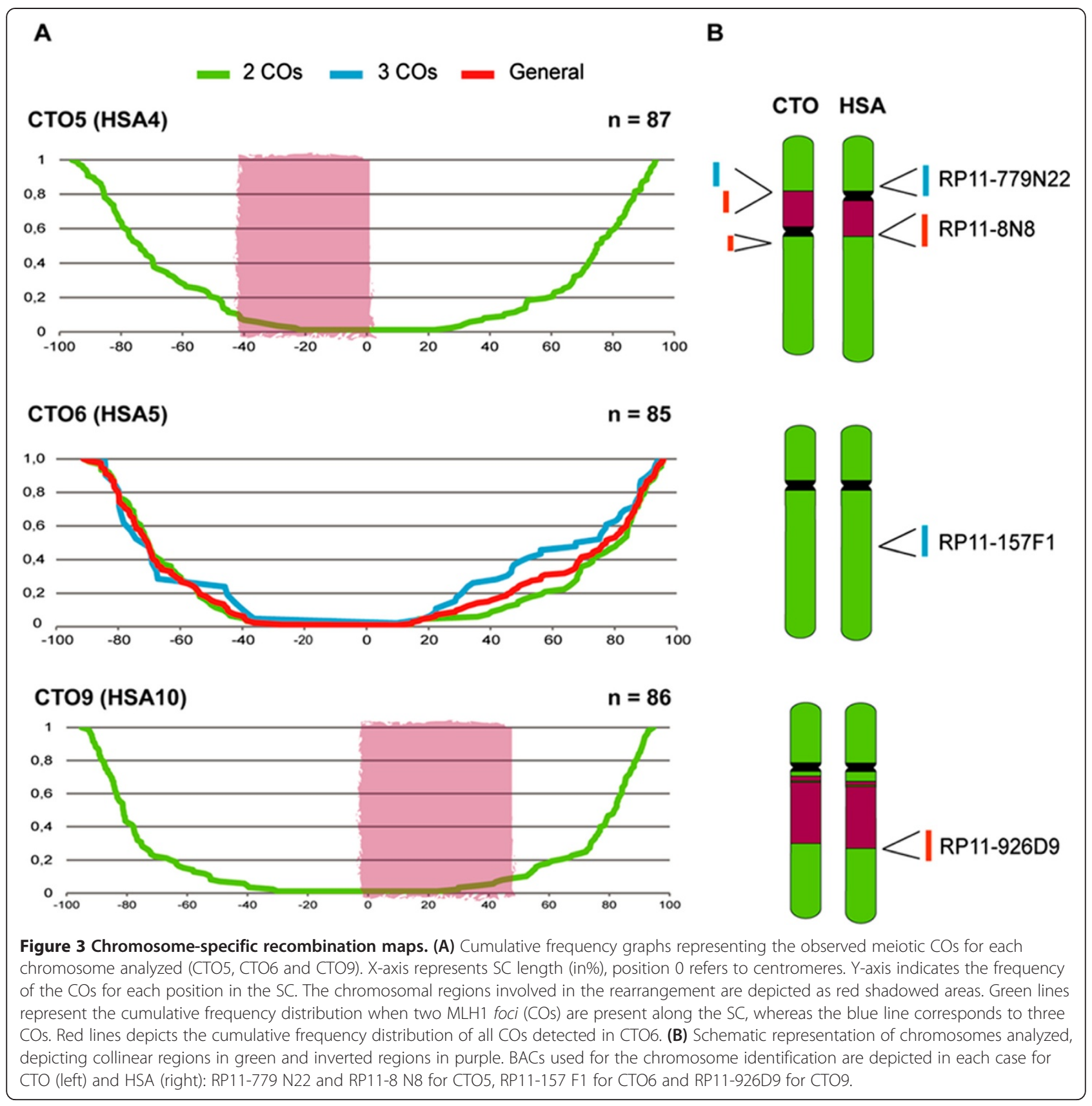

EBRs and HSBs defined in the macaque genome has revealed an interesting pattern: evolutionary breakpoints are gene-rich regions, with a significant functional clustering for genes related to the immune system. Furthermore, and in light of our observations, inversions can induce a reduction in the recombination rate among the different alleles contained in the inversion, which could be favoring adaptive alleles. Future comparative research on the effect of chromosomal reorganization on recombination as outlined above should be an effective means to enhance our knowledge of the role of genome reshuffling in evolution.

\section{Methods}

Whole-genome comparisons and evolutionary breakpoint definition

The experimental design of the study is represented in Figure 1 . In order to detect the evolutionary breakpoint regions (EBRs) and homologous synteny blocks (HSBs) between human and macaque whole-genome sequences, two different algorithms were applied: SyntenyTracker [67] and Cassis [68]. Both approaches compare the order and orientation of orthologous markers (genes) between genomes, detecting changes both in the sequence order and the position of the HSBs and EBRs. Orthologous 
genes between human (GRch37.p7) and rhesus monkey (MMUL_1.0) genomes were obtained through the BioMart database (http://www.ensembl.org/index.html). SyntenyTracker [67] determines the position in the chromosome sequences of both genomes, providing information of the relative orientation of each HSB. This enables the detection of chromosomal rearrangements such as inversions and/or translocations between two genomes. Once the HSBs were detected, genomic regions between consecutive HSBs were considered EBRs. Cassis [68], on the other hand, is especially designed to define breakpoint regions, providing information about different types of rearrangements, such as inversions, translocations or indels. Both algorithms were applied as previously described [5] using default parameters for SyntenyTracker and level 1 in the lastz alignment in Cassis. In order to obtain the genomic positions of EBRs in both genomes, the analysis was performed in two directions: (i) using both the human genome as reference genome, and (ii) the macaque genome as reference. Following previous studies [19,22], we considered EBRs that were $4 \mathrm{Mbp}$ in size or less. Regions larger than $4 \mathrm{Mbp}$ in size were considered "gaps". Furthermore, we labeled as telomeric/subtelomeric the 2 $\mathrm{Mbp}$ at the ends of each human chromosome and as centromeric/pericentromeric the $2 \mathrm{Mbp}$ regions flanking the unknown nucleotides (Ns), as previously described [25].

Once the genomic positions of EBRs were obtained, we followed conservative criteria in order to avoid false positives. To do so, EBRs located at telomeres, centromeres and gaps were excluded from the analysis. The resulting EBRs were classified according to whether they are involved in macro-rearrangements (rearranged regions $>1.4$ $\mathrm{Mbp}$ ) or micro-rearrangements (rearranged regions $<1.4$ Mbp). Simultaneously, we also classified each EBR depending on which type of chromosomal rearrangement was involved, that is, inversion, fusion or fission, following previous studies [30].

\section{Analysis of repetitive elements and gene screening}

The distribution of TR in the macaque genome using the eTandem algorithm was analyzed (part of EMBOSS 6.0.1 [69]). The eTandem algorithm was run with a minimum repeat unit of $2 \mathrm{bp}$ and a maximum repeat unit of $100 \mathrm{bp}$, as previously described [26]. The resulting output files were computed for the detection of overlapping TR, and the canonical motif was defined using in-home Perl scripts. In order to compare the distribution of TR along macaque chromosomes, we counted the base pairs of tandem repeats in $100 \mathrm{Kbp}$ windows for each chromosome. Finally, each window was labeled according to its position: telomere, centromere, HSBs or EBRs. Using Perl scripts, we computed the density of TR and merged the positions of TR with the different types of genomic regions in the human genome.
Then, the number and the genomic position of annotated genes in the macaque genome were considered (RefSeq from the MMUL_1.0 assembly) to perform the gene distribution analysis using the BioMart browser of Ensembl (release 67). We grouped all genes with a known function in non-overlapping windows of $100 \mathrm{Kbp}$ and labeled each window according to its position: telomere, centromere, HSBs or EBRs. In this case, the variable considered was gene count in each window, given that we analyzed presence/absence of genes, rather than the number of base-pairs covered by genes.

The Functional Annotation Clustering tool in DAVID (Database for Annotation, Visualization, and Integrated Discovery, v6.7) [50], was used in order to identify overrepresented biological terms contained in EBRs. Functional annotation clustering allows for the biological interpretation at a 'biological module' level of the most relevant biological terms (GO). Following algorithm's recommendations, all clusters analyzed included a minimum of 10 genes and a maximum of 3,000 [50]. In DAVID annotation system, Fisher Exact is adopted to measure the gene-enrichment in annotation terms by means of an EASE-score, a modified Fisher Exact P-Value. EASEscores equal or smaller than 0.05 were considered statistically significant (i.e., strongly enriched in the annotation categories). Additionally, the system uses the group Enrichment Score (a geometric mean of member's p-values in a corresponding annotation cluster) to rank the biological significance of the genes found in a cluster. Enrichment Scores $\geq 1.5$ indicated significant over-represented of gene functions.

\section{Biological samples}

Metaphase chromosomes were prepared from peripheral blood samples obtained from one female rhesus macaque (Parc Zoològic de Barcelona, Spain). Cultures from peripheral blood samples were processed under standard conditions in order to obtain chromosome preparations as previously described [35]. Additionally, testicular tissue from an adult individual of Cercocebus torquatus (CTO, $2 n=42)$ with proven fertility was used for the study of meiotic recombination. In order to obtain spermatocyte spreads, testicular tissue was processed as previously described $[64,65]$.

\section{Immunofluorescence}

Immunostaining of meiocytes was performed as previously described $[64,65]$. Different sets of antibodies were used: rabbit anti-SYCP3 (Abcam), human anti-CenP (human serum CREST, a kind gift from Dr. M. Fritzel) and mouse anti-MLH1 (Pharmigen) for the detection of meiotic crossovers (COs), all of them diluted in PTBG solution (0.05\% Tween 20 in PBS) 1:200, 1:200 and 1:100, respectively. Fluorochrome-conjugated secondary antibodies (all from 
Jackson Immunoresearch) were used for detection: goat anti-rabbit conjugated with $\mathrm{Cy} 3$, goat anti-human conjugated with $\mathrm{Cy} 5$ and goat anti-mouse conjugated with FITC diluted 1:100 in PTBG.

\section{Fluorescence in situ hybridization}

BAC clones spanning evolutionary breakpoints were obtained from the human library available at CHORI (Children's Hospital Oakland Research Institute) (Additional file 6: Table S5). DNA from BACs was extracted according to standard protocols using a commercial kit (QIAGEN Plasmid). Fluorescence in situ hybridization (FISH) with specific BAC clones was performed on both metaphase chromosomes and spermatocyte spreads as previously described $[40,64]$. Briefly, $1 \mu \mathrm{g}$ of the DNA plasmid was labeled with dUTP-digoxygenine by Nick Translation (Abbot kit) and ethanol precipitated with competitor DNA (Cot-1 human DNA, Invitrogen, $1 \mathrm{mg} / \mathrm{ml}$ ), salmon sperm DNA (Invitrogen, $10 \mathrm{mg} / \mathrm{ml}$ ) and $1 / 10$ volume of $3 \mathrm{~mol} / \mathrm{L}$ sodium acetate overnight at $-20^{\circ} \mathrm{C}$. The precipitated probe mix was resuspended in $14 \mathrm{ml}$ hybridization buffer (50\% deionized formamide, 10\% dextran sulfate, 2xSSC and $0.5 \mathrm{~mol} / \mathrm{L}$ phosphate), denatured $80^{\circ} \mathrm{C}$ for $10 \mathrm{~min}$ and pre-annealed at $37^{\circ} \mathrm{C}$ for $1 \mathrm{~h}$. Preparations were visualized using a Zeiss Axioskop epifluorescence microscope equipped with the appropriate filters and a charged coupled-device camera (ProgRes ${ }^{\oplus}$ CS10plus, Je noptik).

\section{Recombination analysis}

The Micromeasure 3.3 software [70] was used for image analysis and for the construction of chromosome-specific recombination maps based on the relative distances between adjacent MLH1 foci, a marker for meiotic crossovers (COs). For each chromosome analyzed, the position of each MLH1 foci was recorded as a relative position (as the percentage of total length of the synaptonemal complex, SC) from the centromere, identified by the centromeric signal in each preparation as described previously $[64,65]$. Using the centromere as a reference, the positions of each MLH1 focus were calculated along the SC, from the centromere to the telomere. Thus, for comparison among chromosomes, the position of MLH1 foci was expressed as the relative position of each $\mathrm{CO}$ to the length of the chromosome (the length of each SC was divided into $10 \%$ intervals). To convert the MLH1 foci to genetic distances, the number of MLH1 foci detected per SC was multiplied by a factor of 50 map units ( 1 crossover $=$ $50 \mathrm{cM})[64,65]$.

The effect of chromosome inversions on the CO distribution pattern was analyzed by calculating MLH1 foci density within inverted and non-inverted regions, considering the length (expressed in $\mu \mathrm{m}$ ) for each region, so the differences due to $\mathrm{SC}$ lengths for each chromosome were normalized. In order to delimit the inversions directly in spermatocytes, the centromere position and the specific $\mathrm{BAC}$ probes labeling the breakpoint distal to centromere was used (Figure 1 and Additional file 6: Table S5). To allow for comparison among chromosomes, $\mathrm{CO}$ distribution was expressed as the relative position of each $\mathrm{CO}$ to the length of the chromosome (the length of each SC was divided into $10 \%$ intervals).

Furthermore, and in order to disentangle the centromeric effect on recombination, the $\mathrm{CO}$ distribution was compared between the rearranged chromosomes and the collinear one, using the last as a control. To this purpose, we simulated an inversion in the collinear chromosome, by analyzing the recombination pattern of a region of (proportionally) the same size as the observed inverted region. We constructed plots of cumulative frequency to study the pattern along the chromosome arms.

Statistical analysis was performed using JMP 10 software (SAS Institute Inc.) and IBM SPSS Statistics 20, using the Kolmogorov-Smirnov-Lilliefors test for normality, and the Kruskal-Wallis and Mann-Whitney U tests for comparisons.

\section{Additional files}

Additional file 1: Table S1. EBR positions involved in the macrorearrangements (inversions spanning more than $4 \mathrm{Mbp}$, fusions and fissions) between human and macaque genomes detected in our study.

Additional file 2: Table S2. EBR positions involved in the microrearrangements (inversions spanning less than $4 \mathrm{Mbp}$, indels and highcomplex regions) between human and macaque genomes detected in our study.

Additional file 3: Figure S1. Highly refined map of the reorganizations and evolutionary breakpoint regions in the human and rhesus macaque genomes. Representation of HSB (gray blocks) and EBRS (white regions) between human and rhesus monkey, using human as the reference genome detected by SyntenyTracker (Macaque_Synteny) and Cassis (Macaque_Cassis) algorithms, as well as the final model (Macaque_Final) (from left to right in each chromosome representation). The final model is the result of merging the outputs of both programs. Inset numbers represent the homologous rhesus monkey chromosomes. Hatched areas represent heterochromatin in the human genome.

Additional file 4: Table S3. Output from Functional Annotation Tool of DAVID using genes inside EBR positions (+-200 kbp) (see materials and methods for details). Annotation clusters are statistically significant when Enrichment Score is above 1.5

Additional file 5: Table S4. Output from Functional Annotation Tool of DAVID using genes inside the MMU5 (see materials and methods for details). Annotation clusters are statistically significant when Enrichment Score is above 1.5 .

Additional file 6: Table S5. Selection of human BAC clones for the EBRs detected in silico.

\section{Abbreviations}

HSBs: Homologous syntenic blocks; EBRs: Evolutionary breakpoint regions; SYCP3: Synaptonemal complex protein 3; SC: Synaptonemal complex; MLH1: MutL homolog 1; BAC: Bacterial artificial clones; CHORI: Children's Hospital Oakland Research Institute; COs: Crossovers; MMU: Macaca mulatta; CTO: Cercocebus torquatus; TR: Tandem repeats; HSA: Homo sapiens. 


\section{Competing interest}

The authors declare that they have no competing interests.

\section{Authors' contributions}

Conceived and supervised the study: ARH. Performed the experiments: AU, MF and LC. Analyzed the data: AU, MF and ARH. Wrote the paper: AU, MF and $\mathrm{ARH}$. All authors have read and given approval of the final version of the manuscript.

\section{Acknowledgements}

The authors are especially thankful to Hugo Fernandez-Bellón, from the Barcelona Zoo, for his contribution in collecting biological samples. This work was supported by the Ministerio de Economia y Competitividad (CGL-2010-20170).

\section{Author details}

${ }^{1}$ Institut de Biotecnologia i Biomedicina (IBB), Universitat Autònoma de Barcelona, Campus UAB, 08193, Cerdanyola del Vallès, Barcelona, Spain. ${ }^{2}$ Present address: Institut de Biologia Evolutiva (CSIC-Universitat Pompeu Fabra), Passeig Marítim de la Barceloneta 37-49, 08003 Barcelona, Spain. ${ }^{3}$ Departament de Biologia Cel.Jular, Fisiologia i Immunologia, Universitat Autònoma de Barcelona, Campus UAB, 08193, Cerdanyola del Vallès, Barcelona, Spain. ${ }^{4}$ Present address: Department of Comparative Biomedical Sciences, The Royal Veterinary College, Royal College Street, London NW1 OTU, UK.

Received: 24 February 2014 Accepted: 19 June 2014 Published: 26 June 2014

\section{References}

1. Butlin RK: Recombination and speciation. Mol Ecol 2005, 14:2621-2635.

2. Faria R, Navarro A: Chromosomal speciation revisited: rearranging theory with pieces of evidence. Trends Ecol Evol 2010, 25:660-669.

3. Brown JD, O'Neill RJ: Chromosomes, conflict, and epigenetics: chromosomal speciation revisited. Annu Rev Genomics Hum Genet 2010, 11:291-316.

4. Hoffmann AA, Rieseberg LH: Revisiting the impact of inversions in evolution: from population genetic markers to drivers of adaptive shifts and speciation? Annu Rev Ecol Evol Syst 2008, 39:21-42.

5. Farré M, Micheletti $D$, Ruiz-Herrera A: Recombination rates and genomic shuffling in human and chimpanzee-a new twist in the chromosomal speciation theory. Mol Biol Evol 2013, 30:853-864.

6. Noor MAF, Grams KL, Bertucci LA, Reiland J: Chromosomal inversions and the reproductive isolation of species. Proc Natl Acad Sci 2001, 98:12084-12088.

7. Rieseberg LH: Chromosomal rearrangements and speciation. Trends Ecol Evol 2001, 16:351-358.

8. Navarro A, Barton NH: Chromosomal speciation and molecular divergence-accelerated evolution in rearranged chromosomes. Science 2003, 300:321-324.

9. Rieseberg LH, Whitton J, Gardner K: Hybrid zones and the genetic architecture of a barrier to gene flow between two sunflower species. Genetics 1999, 152:713-727.

10. Brown KM, Burk LM, Henagan LM, Noor MAF: A test of the chromosomal rearrangement model of speciation in Drosophila pseudoobscura. Evolution 2004, 58:1856-1860

11. Rieseberg LH, Willis JH: Plant speciation. Science 2007, 317:910-914.

12. Kulathinal RJ, Bennett SM, Fitzpatrick CL, Noor MAF: Fine-scale mapping of recombination rate in Drosophila refines its correlation to diversity and divergence. Proc Natl Acad Sci U S A 2008, 105:10051-10056.

13. Michel AP, Grushko O, Guelbeogo WM, Lobo NF, Sagnon N, Costantini C, Besansky NJ: Divergence with gene flow in Anopheles funestus from the Sudan Savanna of Burkina Faso, West Africa. Genetics 2006, 173:1389-1395.

14. Marques-Bonet T, Sànchez-Ruiz J, Armengol L, Khaja R, Bertranpetit J, Lopez-Bigas N, Rocchi M, Gazave E, Navarro A: On the association between chromosomal rearrangements and genic evolution in humans and chimpanzees. Genome Biol 2007, 8:R230.

15. Franchini $P$, Colangelo $P$, Solano E, Capanna E, Verheyen E, Castiglia R: Reduced gene flow at pericentromeric loci in a hybrid zone involving chromosomal races of the house mouse Mus musculus domesticus. Evolution 2010, 64:2020-2032.

16. Yannic G, Basset $P$, Hausser J: Chromosomal rearrangements and gene flow over time in an inter-specific hybrid zone of the Sorex araneus group. Heredity 2009, 102:616-625.

17. Navarro A, Betrán E, Barbadilla A, Ruiz A: Recombination and gene flux caused by gene conversion and crossing over in inversion heterokaryotypes. Genetics 1997, 146:695-709.

18. Pevzner $P$, Tesler G: Human and mouse genomic sequences reveal extensive breakpoint reuse in mammalian evolution. Proc Natl Acad Sci U S A 2003, 100:7672-7677

19. Larkin DM, Pape G, Donthu R, Auvil L, Welge M, Lewin HA: Breakpoint regions and homologous synteny blocks in chromosomes have different evolutionary histories. Genome Res 2009, 19:770-777.

20. Robinson TJ, Ruiz-Herrera A: Mammalian Chromosomal Evolution: From Ancestral States to Evolutionary Regions. Berlin (Germany): Springer; 2010:143-158.

21. Ruiz-Herrera A, Farré M, Robinson TJ: Molecular cytogenetic and genomic insights into chromosomal evolution. Heredity 2012, 108:28-36.

22. Murphy WJ, Larkin DM, Everts-van der Wind A, Bourque G, Tesler G, Auvil L, Beever JE, Chowdhary BP, Galibert F, Gatzke L, Hitte C, Meyers SN, Milan D, Ostrander EA, Pape G, Parker HG, Raudsepp T, Rogatcheva MB, Schook LB, Skow LC, Welge M, Womack JE, O'brien SJ, Pevzner PA, Lewin HA: Dynamics of mammalian chromosome evolution inferred from multispecies comparative maps. Science 2005, 309:613-617.

23. Bourque G, Pevzner PA, Tesler G: Reconstructing the genomic architecture of ancestral mammals: lessons from human, mouse, and rat genomes. Genome Res 2004, 14:507-516.

24. Ruiz-Herrera A, García F, Giulotto E, Attolini C, Egozcue J, Ponsà M, Garcia M: Evolutionary breakpoints are co-localized with fragile sites and intrachromosomal telomeric sequences in primates. Cytogenet Genome Res 2005, 108:234-247.

25. Ruiz-Herrera A, Castresana J, Robinson TJ: Is mammalian chromosomal evolution driven by regions of genome fragility? Genome Biol 2006, 7:R115.

26. Farré M, Bosch M, López-Giráldez F, Ponsà M, Ruiz-Herrera A: Assessing the role of tandem repeats in shaping the genomic architecture of great apes. Plos One 2011, 6:e27239.

27. Robinson TJ, Ruiz-Herrera A, Froenicke L: Dissecting the mammalian genome-new insights into chromosomal evolution. Trends Genet 2006, 22:297-301

28. Kemkemer C, Kohn M, Cooper DN, Froenicke L, Högel J, Hameister H, Kehrer-Sawatzki H: Gene synteny comparisons between different vertebrates provide new insights into breakage and fusion events during mammalian karyotype evolution. BMC Evol Biol 2009, 9:84.

29. Longo MS, Carone DM, Green ED, O'Neill MJ, O'Neill RJ: Distinct retroelement classes define evolutionary breakpoints demarcating sites of evolutionary novelty. BMC Genomics 2009, 10:334.

30. Girirajan S, Chen L, Graves T, Marques-Bonet T, Ventura M, Fronick C, Fulton L, Rocchi M, Fulton RS, Wilson RK, Mardis ER, Eichler EE: Sequencing human-gibbon breakpoints of synteny reveals mosaic new insertions at rearrangement sites. Genome Res 2009, 19:178-190.

31. Mlynarski EE, Obergfell CJ, O'Neill MJ, O'Neill RJ: Divergent patterns of breakpoint reuse in Muroid rodents. Mamm Genome 2010, 21:77-87.

32. Lemaitre C, Zaghloul L, Sagot M-F, Gautier C, Arneodo A, Tannier E, Audit B: Analysis of fine-scale mammalian evolutionary breakpoints provides new insight into their relation to genome organisation. BMC Genomics 2009, 10:335.

33. Muñoz A, Sankoff D: Detection of gene expression changes at chromosomal rearrangement breakpoints in evolution. BMC Bioinformatics 2012, 13(Suppl 3):S6.

34. Müller S, Wienberg J: "Bar-coding" primate chromosomes: molecular cytogenetic screening for the ancestral hominoid karyotype. Hum Genet 2001, 109:85-94.

35. Ruiz-Herrera A, Ponsà M, García F, Egozcue J, García M: Fragile sites in human and Macaca fascicularis chromosomes are breakpoints in chromosome evolution. Chromosome Res 2002, 10:33-44.

36. Gibbs RA, Rogers J, Katze MG, Bumgarner R, Weinstock GM, Mardis ER, Remington KA, Strausberg RL, Venter JC, Wilson RK, Batzer MA, Bustamante CD, Eichler EE, Hahn MW, Hardison RC, Makova KD, Miller W, Milosavljevic A, Palermo RE, Siepel A, Sikela JM, Attaway T, Bell S, Bernard KE, Buhay CJ, Chandrabose MN, Dao M, Davis C, Delehaunty KD, Ding Y, et al: Evolutionary and biomedical insights from the rhesus macaque genome. Science 2007, 316:222-234. 
37. Ventura M, Antonacci F, Cardone MF, Stanyon R, D'Addabbo P, Cellamare A, Sprague $L$, Eichler EE, Archidiacono N, Rocchi M: Evolutionary formation of new centromeres in macaque. Science 2007, 316:243-246.

38. Stanyon R, Rocchi M, Capozzi O, Roberto R, Misceo D, Ventura M, Cardone MF, Bigoni F, Archidiacono N: Primate chromosome evolution: ancestral karyotypes, marker order and neocentromeres. Chromosome Res 2008, 16:17-39.

39. De Oliveira EHC, Neusser M, Müller S: Chromosome evolution in new world monkeys (Platyrrhini). Cytogenet Genome Res 2012, 137:259-272.

40. Ruiz-Herrera A, Garcia F, Frönicke L, Ponsà M, Egozcue J, Caldés MG, Stanyon R: Conservation of aphidicolin-induced fragile sites in Papionini (Primates) species and humans. Chromosome Res 2004, 12:683-690.

41. Bailey JA, Eichler EE: Primate segmental duplications: crucibles of evolution, diversity and disease. Nat Rev Genet 2006, 7:552-564.

42. Kehrer-Sawatzki H, Sandig CA, Goidts V, Hameister H: Breakpoint analysis of the pericentric inversion between chimpanzee chromosome 10 and the homologous chromosome 12 in humans. Cytogenet Genome Res 2005, 108:91-97.

43. Zhao H, Bourque $\mathrm{G}$ : Recovering genome rearrangements in the mammalian phylogeny. Genome Res 2009, 19:934-942.

44. Carbone L, Harris RA, Vessere GM, Mootnick AR, Humphray S, Rogers J, Kim SK, Wall JD, Martin D, Jurka J, Milosavljevic A, de Jong PJ: Evolutionary breakpoints in the gibbon suggest association between cytosine methylation and karyotype evolution. PLoS Genet 2009, 5:e1000538.

45. Bourque G: Transposable elements in gene regulation and in the evolution of vertebrate genomes. Curr Opin Genet Dev 2009, 19:607-612.

46. Delprat A, Negre B, Puig M, Ruiz A: The transposon Galileo generates natural chromosomal inversions in Drosophila by ectopic recombination. PLoS One 2009, 4:e7883.

47. Karere GM, Froenicke L, Millon L, Womack JE, Lyons LA: A high-resolution radiation hybrid map of rhesus macaque chromosome 5 identifies rearrangements in the genome assembly. Genomics 2008, 92:210-218.

48. Zhang X, Goodsell J, Norgren RB: Limitations of the rhesus macaque draft genome assembly and annotation. BMC Genomics 2012, 13:206.

49. Groenen MAM, Archibald AL, Uenishi H, Tuggle CK, Takeuchi Y, Rothschild MF, Rogel-Gaillard C, Park C, Milan D, Megens H-J, Li S, Larkin DM, Kim H, Frantz LAF, Caccamo M, Ahn H, Aken BL, Anselmo A, Anthon C, Auvil L, Badaoui B, Beattie CW, Bendixen C, Berman D, Blecha F, Blomberg J, Bolund L, Bosse M, Botti S, Bujie Z, et al: Analyses of pig genomes provide insight into porcine demography and evolution. Nature 2012, 491:393-398.

50. Huang DW, Sherman BT, Lempicki RA: Systematic and integrative analysis of large gene lists using DAVID bioinformatics resources. Nat Protoc 2009, 4:44-57.

51. Yamaguchi $Y$, Ouchi $Y$ : Antimicrobial peptide defensin: identification of novel isoforms and the characterization of their physiological roles and their significance in the pathogenesis of diseases. Proc Jpn Acad Ser B Phys Biol Sci 2012, 88:152-166.

52. Radhakrishnan Y, Hamil KG, Yenugu S, Young SL, French FS, Hall SH: Identification, characterization, and evolution of a primate beta-defensin gene cluster. Genes Immun 2005, 6:203-210

53. Semple CA, Gautier P, Taylor K, Dorin JR: The changing of the guard: Molecular diversity and rapid evolution of beta-defensins. Mol Divers 2006, 10:575-584.

54. Patil AA, Cai Y, Sang Y, Blecha F, Zhang G: Cross-species analysis of the mammalian beta-defensin gene family: presence of syntenic gene clusters and preferential expression in the male reproductive tract. Physiol Genomics 2005, 23:5-17.

55. Elsik CG, Tellam RL, Worley KC, Gibbs RA, Muzny DM, Weinstock GM, Adelson DL, Eichler EE, Elnitski L, Guigó R, Hamernik DL, Kappes SM, Lewin HA, Lynn DJ, Nicholas FW, Reymond A, Rijnkels M, Skow LC, Zdobnov EM, Schook L, Womack J, Alioto T, Antonarakis SE, Astashyn A, Chapple CE, Chen H-C, Chrast J, Câmara F, Ermolaeva O, Henrichsen CN, et al: The genome sequence of taurine cattle: a window to ruminant biology and evolution. Science 2009, 324:522-528.

56. Rostène W, Dansereau M-A, Godefroy D, Van Steenwinckel J, Reaux-Le Goazigo A, Mélik-Parsadaniantz S, Apartis E, Hunot S, Beaudet N, Sarret P: Neurochemokines: a menage a trois providing new insights on the functions of chemokines in the central nervous system. J Neurochem 2011, 118:680-694.
57. Ragozzino D: CXC chemokine receptors in the central nervous system: Role in cerebellar neuromodulation and development. J Neuroviro/ 2002, 8:559-572.

58. Abildskov K, Weldy P, Garland M: Molecular cloning of the baboon UDP-glucuronosyltransferase $2 \mathrm{~B}$ gene family and their activity in conjugating morphine. Drug Metab Dispos 2010, 38:545-553.

59. Lindblad-Toh K, Garber M, Zuk O, Lin MF, Parker BJ, Washietl S, Kheradpour P, Ernst J, Jordan G, Mauceli E, Ward LD, Lowe CB, Holloway AK, Clamp M, Gnerre S, Alföldi J, Beal K, Chang J, Clawson H, Cuff J, Di Palma F, Fitzgerald S, Flicek P, Guttman M, Hubisz MJ, Jaffe DB, Jungreis I, Kent WJ, Kostka D, Lara $M$, et al: A high-resolution map of human evolutionary constraint using 29 mammals. Nature 2011, 478:476-482.

60. Ruiz-Herrera A, Farré M, Ponsà M, Robinson TJ: Selection against Robertsonian fusions involving housekeeping genes in the house mouse: integrating data from gene expression arrays and chromosome evolution. Chromosome Res 2010, 18:801-808.

61. Hassold T, Hansen T, Hunt P, VandeVoort C: Cytological studies of recombination in rhesus males. Cytogenet Genome Res 2009, 124:132-138.

62. Ponsà M, Estop AM, Miró R, Rubio A, Egozcue J: Banding patterns of the chromosomes of Miopithecus talapoin compared with Macaca mulatta and Cercopithecus aethiops. Cytogenet Cell Genet 1980, 28:41-46.

63. Brown CJ, Dunbar VG, Shafer DA: A comparison of the karyotypes of six species of the genus Macaca and a species of the genus Cercocebus. Folia Primatol (Basel) 1986, 46:164-172.

64. Garcia-Cruz R, Pacheco S, Brieño MA, Steinberg ER, Mudry MD, Ruiz-Herrera A, Garcia-Caldés M: A comparative study of the recombination pattern in three species of Platyrrhini monkeys (Primates). Chromosoma 2011, 120:521-530.

65. Segura J, Ferretti L, Ramos-Onsins S, Capilla L, Farré M, Reis F, Oliver-Bonet M, Fernández-Bellón H, Garcia F, Garcia-Caldés M, Robinson TJ, Ruiz-Herrera A: Evolution of recombination in eutherian mammals: insights into mechanisms that affect recombination rates and crossover interference. Proc Biol Sci 2013, 280:20131945.

66. Froenicke L, Anderson LK, Wienberg J, Ashley T: Male mouse recombination maps for each autosome identified by chromosome painting. Am J Hum Genet 2002, 71:1353-1368.

67. Donthu R, Lewin HA, Larkin DM: SyntenyTracker: a tool for defining homologous synteny blocks using radiation hybrid maps and whole-genome sequence. BMC Res Notes 2009, 2:148.

68. Baudet C, Lemaitre C, Dias Z, Gautier C, Tannier E, Sagot M-F: Cassis: detection of genomic rearrangement breakpoints. Bioinformatics 2010, 26:1897-1898

69. Rice P, Longden I, Bleasby A: EMBOSS: the European Molecular Biology Open Software Suite. Trends Genet 2000, 16:276-277.

70. Reeves A: MicroMeasure: a new computer program for the collection and analysis of cytogenetic data. Genome 2001, 44:439-443.

doi:10.1186/1471-2164-15-530

Cite this article as: Ullastres et al:: Unraveling the effect of genomic structural changes in the rhesus macaque - implications for the adaptive role of inversions. BMC Genomics 2014 15:530.

\section{Submit your next manuscript to BioMed Central and take full advantage of:}

- Convenient online submission

- Thorough peer review

- No space constraints or color figure charges

- Immediate publication on acceptance

- Inclusion in PubMed, CAS, Scopus and Google Scholar

- Research which is freely available for redistribution 